\title{
El concepto español de cabeza de familia
}

\author{
I \\ I N T R O D U C C I Ó N
}

El concepto orgánico de la población en que se funda el Nuevo Estado ha sido la causa de que la participación del pueblo en sus tareas se haga a través de tres instituciones: Familia, Municipio y Sindicato. Consecuencia lógica de este concepto es que la elección de los concejales, según la reciente Ley de Bases de Régimen Local (I), se haga por los vecinos cabezas de familia y por los sindicatos (2), de donde surge, a su vez, la necesidad del Censo Elec- toral de Cabezas de Familia, que ha llevado a cabo el "Instituto Nacional de Estadística".

La formación de este Censo, y su conservación, ha de tener como eje la fijación del concepto de cabeza de familia, concepto más complicado de lo que a primera vista puede parecernos, y cuyo estudio es hoy altamente interesante, tanto para los Municipios españoles como para el "Instituto Nacional de Estadística".

Con un simple análisis de la cuestión, el problema del concepto del cabeza de familia podría parecer resuelto aplicando el artícu-

(1) Vid. la Base VIII.

(2) Mediante el sistema indicado damos cumplimiento a lo ordenado por la doctrina: Familia. Municipio y Sindicato entran en la liza electoral. La tradición española avala el sistema que queremos implantar: el voto del hogar o afogaro, el voto de los gremios y el de selección de propuesta de las Municipalidades libres valencianas son pruebas de lo que acabamos de afirmar.» Del discurso pronunciado en las Cortes por el Excmo. Sr. Ministro de la Gobernación). 
lo $5 .^{\circ}$ del Decreto de 29 de septiembre de 1945, pero, a poco que meditemos, se notará que no cabe una solución tan simplista, por varias razones: primera, porque el citado artículo nos da el concepto de cabeza de familia a los fines puramente electorales, y hay que tener en cuenta que dicho concepto - como luego veremosexistía con anterioridad y respondiendo a otros fines que los propios del sufragio; segunda, porque en el artículo se condiciona el ser o no cabeza de familia a estar inscrito como tal en el Padrón municipal de 1940 y sus rectificaciones, hechas con anterioridad al Decreto, y, por tanto, con arreglo a normas distintas, y tercera, porque -como ha podido apreciarse en la elaboración del Censo- el concepto dado por el Decreto dista mucho de ser perfecto, y su aplicación ha dado lugar, en la práctica, a dificultades para cuya solución conviene tener criterios fijos, con objeto de poder resolver con equidad, en cada caso, el otorgamiento o no del derecho de sufragio, o, lo que es lo mismo, quien es o no cabeza de familia a los fines electorales.

Por tanto, si queremos dar un concepto exacto de cabeza de familia, no debemos conformarnos con una aplicación del fijado en el Decreto, sino estudiar a fondo el concepto, investigar las razones a que obedeció su aparición y las razones prácticas a que en la vida político-administrativa responde, $y$, de esta manera, podremos extraer, con independencia de los distintos criterios que en las leyes se han dado para su determinación, el concepto que consideremos más útil a la ciencia y la práctica administrativa.

Con este propósito, hemos reálizado el presente trabajo, en el que estudiaremos, primero, el origen y evolución del concepto de cabeza de familia, y, después, su concepto en la legislación española vigente y examen crítico de la misma, terminando con unos criterios prácticos para su determinación.

Con ello habremos contribuido, en la medida de nuestras fuerzas, al estudio de un problema tan interesante en los momentos actuales, y si en algo pudiera contribuir este trabajo a aclarar dudas o suscitar interés por el estudio de estas cuestiones, ello sería nuestra mejor recompensa y más intima satisfacción. 


\section{ORIGEN Y EVOLUCión HISTÓRICA DEL CONCEPTO}

I) El concepto de cabeza de familia en la tradición juridica española.

La palabra "cabeza", además de su acepción de "parte del organismo", se emplea también para designar al que rige, guía o gobierna una comunidad social, pública o privada. En este sentido, el Fuero Juzgo y las Partidas (3), consideraban ya a los príncipes y al Rey como "cabezas del Reino", y en este sentido se habla también del Sumo Pontífice como "cabeza visible" de la Iglesia. Es, pues, perfectamente lógico que al que rige, guía o gobierna la sociedad primaria y célula de todas las demás, se le designe también con el nombre de "cabeza de familia" y que, por tanto, siempre que se haya considerado a la familia como institución jurídica con derechos y deberes propios, haya surgido necesariamente el concepto de cabeza de familia, bien con esta denominación o bien con otra distinta.

Sin embargo, la expresión "cabeza de familia" es relativamente reciente en nuestra Patria. Si consultamos el Diccionario Escriche (4), o la Enciclopedia Española de Derecho Administrativo (5), o el Diccionario de Derecho Administrativo español (6), en ninguno de ellos la encontramos. Nos hablan, eso sí, de "cabezas de casa o de linaje", entendiendo por tales los que por "legítima descendencia del fundador tienen primogenitura o heredan sus derechos", pero no mencionan a los cabezas de familia, ni tienen idea de este concepto que hoy nos es $\tan$ conocido y que usamos tan corrientemen-

(3) Fuero Juzgo, Ley IV, tit. I, lib. II: APor ende, debemos primeramente ordenar los fechos de los príncipes, porque son nuestras cabezas.»

(4) ESCRICBE: Diccionario razonado de Legislación y Jurisprudencia, Madrid, año 1838.

(5) Lorenzo Arrazola, Sainz de Andino y ottos: Enciclopedia Española de Derecho y Administración, Madrid, 1852.

(6) Cos- Gayón y Cánovas del Castillo: Diccionario de Derecho Administraıivo Español. 
te (7). Tampoco encontramos la expresión en el famoso índice de Magro y Zurita (8), ni en la Novísima Recopilación. Parece extraño que en nuestra tradición jurídica no exista el concepto de cabeza de familia, ya que, por ser España un país de honda y ferviente tradición católica, la familia ocupó siempre el rango social que en los pueblos cristianos le corresponde. Pero esta extrañeza desaparece si tenemos en cuenta que el concepto de cabeza de familia estuvo incluído o absorbido por otros dos, según su esfera: el de "pater familias" en lo civil y el de vecino a los efectos administrativos de la vida municipal.

En efecto, por vecino entendían nuestros antiguos Ayuntamientos el que era jefe de una casa abierta en el término municipal, gozaba de los derechos del procomún y contribuía al sostenimiento de las cargas municipales. Fueron las leyes municipales del siglo xIX, especialmente las más influídas por el liberalismo, las que extendieron el concepto de vecino a todos los residentes mayores de edad o emancipados, adulterando así el concepto tradicional de vecino.

Este concepto tradicional de vecino, a que venimos refiriéndonos, podemos verlo reflejado en nuestras leyes antiguas, en las viejas Ordenanzas municipales y en los tratadistas de Derecho municipal anteriores al siglo XIX.

Las Partidas exigían diez años de residencia para obtener la cualidad de vecino (9), y la Novísima Recopilación (10) otorgaba et título de vecino "a los que demuestran tal ánimo, como si uno ven-

(7) La Enciclopedia Española de Derecho Administrativo, de Arrazola, en a artículo Ayuntamiento», al comentar el artículo 13 de la Ley Municipal de 1845, da la siguiente definición del concepto de cabeza de familia: aLa persona que se halla al frente de una casa o familia, con las obligaciones y derechos civiles consiguientes, Y tiene el cargo de dirigir y gobernar los negocios de la misma, para lo cual está comprendido como tal en los padrones formados por la autoridad competente.. Dicho artículo de esta Enciclopedia contiene además comentarios muy extensos e interesantes sobre el cabeza de familia, interés que se acentúa si se tiene en cuenta que están escritos antes de que el concepto de cabeza de familia apareciese a efectos estadisticos y en las Leyes municipales.

(8) Magro y Zurita: Indice de proposiciones de las Leyes de la Recopilación... hasta el año 17\%4, Alcalá, 1726.

(9) Código de las Siete Partidas: Ley XXXII, tít. II, parte III ; Ley II, títu. lo XXIV, parte IV.

(10) Novisima Recopilación: Ley VI, tit. IV, lib. 7. 
diese sus bienes en un punto y los comprase en otro adonde se hubiera trasladado. El vecino debía, pues, estar "afincado" en el término municipal.

En las Ordenanzas municipales antiguas como, por ejemplo, las de Canseco (reformadas en I76I (II), se excluía a los mozos solteros de los Concejos de vecinos, y el sentido tradicional era en nuestros pueblos, y lo es aún en muchos de ellos, que cada vecino representaba un hogar o "fogar" (12). Como afirma Pedregal (13), refiriéndose a nuestros pueblos asturianos, "para los efectos de la vida local, no son vecinos los que tienen ese carácter con arreglo a la Ley municipal. Es vecino el que puede cortar, rozar, cavar, cerrar, usar y aprovechar los pastos de los terrenos comunes para sus ganados; y todos esos derechos, con los deberes correlativos, van inherentes a la propiedad colectiva; no depende exclusivamente de la voluntad del recién llegado adquirir la cualidad de vecino".

En nuestros tratadistas anteriores al siglo xix encontramos algunos, como Santayana Bustillo, cuya autoridad y méritos son bien conocidos de todos los municipalistas españoles, que nos dan el siguiente concepto de vecino: "diráse vecino... el que en el pueblo tiene establecida su familia, casa y lo mejor de su hacienda" (14).

Finalmente, la confirmación de lo que venimos sosteniendo, respecto a que el concepto de cabeza de familia se hallaba absorbido en la tradición jurídica española por el concepto de vecino, la encontramos en el artículo I 3 de la Ley municipal del año i845, que dice: "se consideran vecinos para los efectos de esta ley, todos los que siendo cabezas de familia con casa abierta, tengan además un año y un día de residencia o hayan obtenido vecindad con arreglo a las leyes". Es decir, que, según esta ley, para ser vecino era preciso ser cabeza de familia. Por tanto, concluímos: el concepto tradicional español de vecino absorbía el de cabeza de familia, y esto

(11) Costa, Unamuno, G. Linares, Pedregal y otros: Derecho consuetudinario de España, tomo II, pág. 270 ; artículo de D. Elías López Morán: Otrosi, que los mozos solteros no puedan entrar en los Concejos y Juntas de vecinos, pena media cántara de vino..

(12) Véase la nota 2.

(13) Derecho conswetudinario de España, tomo II, pág. 111.

(14) Gobierno politico de los pueblos de España, pág. 109 ; Zaragoza. 1742. 
quizá sea la explicación del por qué no aparece la expresión en nuestra literatura jurídica anterior a las leyes del siglo $\mathrm{xIx}$, las cuales extendieron el concepto del vecino a todos los emancipados.

El concepto de cabeza de familia surgió, pues, en el siglo xix', primero a efectos estadísticos, civiles y administrativos, y posteriormente, ya en el siglo $\mathrm{xx}$, en la legislación municipal, como categoria de la población.

Por interesar hoy principalmente a la Estadística y a los Municipios el nacimiento y la evolución del concepto de cabeza de familia, estudiaremos su origen en las dos esferas.

\section{2) Creación del concepto de cabeza de familia a efectos esta- disticos.}

La Estadística oficial española puede ufanarse de haber formulado el concepto de cabeza de familia treinta años antes de que apareciese en los proyectos de legislación municipal y cincuenta años antes de que fuese una realidad en el Estatuto Municipal de I924.

En 1857, se realizó por la Estadística española la primera inscripción censal, pero tanto en esta inscripción como en la de 1860 no aparece aún el concepto de familia ni el de cabeza de familia, porque la inscripción no se realizaba por familias sino por casas. Así, en las Instrucciones para realización de los Censos de 1857 y de I860 (15), se vislumbra el concepto de cabeza de familia, pero no se lo define. Nos hablan estas Instrucciones de que, "todos los habitantes sin excepción, así nacionales como extranjeros, se empadronarán en la casa o paraje en que pernocten"; de que "las cédulas se llenarán por los cabezas de casa o jefes de establecimiento" (16), etc. No se habla en ningún caso de inscripción por familias. Las cédulas se distribuirán a los vecinos, y cada agente debía señalar el número de casas o habitaciones en donde debia entregar las cédulas (I 7).

(15) De 14 de marzo de 1857 y de 10 de noviembre de 1860 , respectivamente.

(16) Artículo 27 de la Instrucción de 1857.

(17) Artículos 15 y 16 de la Instrucción de 1860. 
Según el Real Decreto de 30 de septiembre de 1858 , la inscripción censal debia verificarse cada cinco años, pero la de 1865 no pudo llevarse a efecto ( 18 ), y el 30 de noviembre de 1864 se dictó un Decreto aplazando la inscripción censal hasta 1870 y ordenando que en lo sucesivo se verificara cada diez años.

En I87o, bajo el gobierno de Serrano y Prim, se publicó un Decreto (7 de junio) dictando disposiciones para el nuevo Censo de población, el cual no llegó a realizarse. No obstante, este Decreto tiene una importancia fundamental para la evolución del concepto de cabeza de familia que venimos estudiando, porque en él se establece por primera vez la inscripción familiar. En la exposición de motivos se hace referencia a las inscripciones de 1857 y 1860 , afirmándose que fueron los primeros pasos "fecundos... pero no tanto como conviniera para llegar al fin deseado". "Considera oportuno el Ministro que suscribe -dice la exposición de motivos-, conformándose con la opinión de la Junta General de Estadística, limitar la investigación a los datos y noticias fundamentales, sin perder de vista los adelantos de la ciencia y los acuerdos de los Congresos internacionales de Estadística." "Las innovaciones que se proponen esto, no obstante, no son escasas." "El sistema de inscripción nominal simultánea, aconsejado por los estadistas (19) y seguido en las dos obras censales de que se ha hecho mérito, continuará aplicándose con una ampliación importante." "Las cédulas permiten anotar por hogares $y$ familias a cuantos individuos, sea cual fuere su condición relativamente al domicilio, se encuentren en un punto dado en el momento del recuento." De acuerdo con esto, el artículo $4^{\circ}$ del citado Decreto establecía que la inscripción se haría por medio de hojas "comprensivas del hogar y la familia" (20).

(18) Según la exposición de motivos del Real decreto de 1864, no se llevó a efecto esta inscripción por estimarse que el nuevo Censo, ssobre multiplicar inmensamente los gastos, no produciria datos más exactos que los anteriores, cuando los pueblos que deben suministrarlos, todavia fatigados con las últimas investigaciones, apenas tendrian aliento para emprender otras nuevaso.

(19) Debe querer decir estadísticos.

(20) El 7 de diciembre de 1870 se dictó por el Ministerio de Hacienda una Orden sustituyendo la cédula de vecindad por la de empadronamiento, y obligandó a todos los acabezas de familian, varones o hembras, mayores de catorce años, con tienes propios y que no fueran pobres de solemnidad, a su adquisición. 
Se modificaba, pues, el sistema de inscripción, adoptándose la cédula familiar, lo que traería como consecuencia la determinación de la familia y del cabeza de la misma.

Aunque la inscripción no se realizó, el paso estaba dado, y en $\mathbf{1} 877$, al ordenarse por Decreto de $\mathbf{I}^{\circ}$ de noviembre una nueva inscripción censal, se reguló que tendría lugar por medio de cédulas duplicadas para cada familia (2I). El concepto de familia, que necesariamente había de surgir de esta forma de inscripción, nos lo da la Instrucción de 1877 al decir en su artículo 16 que "los agentes repartirian las cédulas entregando una por cada familia; y, por consiguiente, cuando vivan reunidos o en compañía individuos, sean parientes, sean extraños, que constituyan familias independientes por contar con recursos propios y atender aisladamente a su sostenimiento, sin que puedan considerarse como huéspedes ni como dependientes unos de otros, reciban tantas cédulas como familias compongan. Así figurarán en cédula aparte los hijos que hayan salido de la patria potestad aunque continúen viviendo al lado de sus padres, si han constituído familia, y los criados casados que tengan familia avecindada dentro del mismo término en que ellos se hallan sirviendo."

Por primera vez se formula aquí, aunque con cierta imprecisión, por la Estadística española, el concepto de familia, del que se deducirá el de cabeza. Este concepto había de perfeccionarse en la inscripción de I 887 para ofrecernos, ya en I900, la siguiente definición del cabeza de familia, según el artículo 20 de la Instrucción para realizar el censo del mismo año. "Se entenderá por cabeza de familia toda persona que estando emancipada cuenta con recursos propios y atiende aisladamente a su sostenimiento y al de sus deudos e hijos si los tuviere."

Ahora bien, ¿a qué razones obedeció el cambio en la forma de la inscripción censal? En primer lugar, como se desprende de los párrafos de la exposición de motivos que hemos transcrito, a razones científicas y técnicas que la Estadística española aconsejaba al compás de los adelantos de la ciencia. Pero, además, había otra razón que se expone en el prólogo al Censo de 1877 por la entonces Di-

(21) También se admitian por primera vez las cédulas azules para las colecti. vidades. 
rección General del Instituto Geográfico y Estadístico. Esta razón era que la clasificación de la población vecinal, tal y como la establecía la Ley municipal de 1870 , entonces vigente (2 I bis), no interesaba a los efectos censales, y era de muy difícil determinación. En efecto, ocurría con frecuencia, que no se hacían las rectificaciones anuales del Padrón y que muchos que debían ser vecinos no se hallaban inscritos. Por otra parte, como el criterio legal de la distinción entre vecinos y domiciliados era el de la emancipación, unas Juntas entendieron que todo español, varón o hembra emancipados, debía ser clasificado como vecino; otras admitieron sólo como emancipación el matrimonio; unas y otras diferían en si la mujer casada debía o no ser considerada como vecina, y otras, finalmente, entendieron que la palabra vecino era inaplicable al concepto de cabeza de familia que se necesitaba a los efectos de la inscripción.

He aquí cómo surgió la necesidad del concepto de cabeza de familia, independientemente del de vecino y del de domiciliado y por insuficiencia de ambos (22).

Pero hay aún algo más importante, y que coincide con la tesis que antes exponíamos, de que el tradicional concepto español de vecino había sido adulterado por las leyes municipales del siglo XIX. Dice así la Dirección General de Estadística en el prólogo del Censo de r877: "En el antiguo organismo de nuestros Ayuntamientos, et jefe de familia con casa abierta, con medios de subsistencia propios y contribuyente del presupuesto municipal, gozaba de los beneficios del procomún con exclusión de los demás habitantes. Tales diferencias fueron disminuyendo, y se llegó a mirar al hijo mayor de edad igual al padre en cuya compañía sigue viviendo sin haber constituído

(21 bis) Según la Ley de 20 de agosto de 1870, era vecino atodo español emancipado que reside habitualmente en el término municipal y se halle inscrito con tal carácter en el padrón del Ayuntamientos, y domiciliado, el que reíne las mismas condiciones, exceptuando la emancipacións.

(22) Con razón decía el entonces Director general de Estadistica en el prólogo al Censo de Población de 1877: aLa primera división que la Ley hace de los habitantes en residentes y transeuntes debe ser en los censos sucesivos la única distinción respecto al domicilio, prescindiendo de la subdivisión en vecinos y domiciliaduss.

Este era también el criterio aceptado en el extranjero, ya que la palabra residente sbarca tanto a los vecinos como a los domiciliados. 
nueva familia. Ya no se exigía para ser vecino, la cualidad de cabeza de familia (23), ni la de contribuir al presupuesto. Pero la opinión vulgar mantiene otro criterio y juzga que cada vecino representa un hogar."

Caló hondo, como vemos, en este problema la Estadística oficial, y bien claro se ve cuán compenetrados estaban sus funcionarios con la tradición jurídica de la vida municipal española.

3) Creación del cabeza de familia en la legislación municipal es-
pañola.

En la esfera municipal aparece con bastante posterioridad el concepto de cabeza de familia. El primer intento de crearlo como categoria jurídica en la clasificación de los habitantes se debió al proyecto de Maura de 1907 (art. 10). Dicho proyecto, que definía el Municipio como "una asociación de familias y casas", definía también al cabeza de familia como el jefe de casa, mayor de edad "bajo cuya dependencia en algún modo viven los individuos de la casa" (art. I I).

Tanto el concepto de familia, así formulado, como la definición de Municipio, antes citada, fueron objeto de dura oposición por la minoría liberal que veía en ellos conceptos nuevos e innecesarios, de carácter reaccionario y contrarios a la libertad e individualidad de la persona humana.

El señor Maldonado, que defendió el dictamen por la Comisión, afirmó que se decía que el Municipio era una asociación de familias, porque la familia representaba el elemento personal del Municipio; "la familia es -decía - la célula social, el único elemento germinativo del Estado, y tenemos forzosamente que decir reunión de familias, porque la asociación de familias constituye el Municipio, como la unión de individuos constituye la familia" (24).

La categoría de cabeza de familia se estimó también por la oposición como "una innovación innecesaria", sin precedentes en nuestra legislación, pero la Comisión la defendió como una consecuencia

(23) Recuérdese lo dispuesto en el artículo 13 de la Ley Municipal de 1845.

(24) Diario de Sesiones del Congreso de los Diputados, sesión del 8 de enero de 1908. pág. 4.230. 
del concepto, ya aprobado, del Municipio, y "porque era necesario para poner en relación la legislación de la vida municipal con el resto de la legislación, pues, empezando por el Código civil en su artículo 1910... y terminando por todas las leyes administrativas y fiscales, el cabeza de familia aparece constantemente, el cabeza de familia está en la vida municipal de hecho, y era necesario llevarlo también a este proyecto para que estuviera regulada de derecho esta categoría importantísima de los habitantes de una población" (25).

Efectivamente, en aquella época la ley del Jurado de 1888 exigía ya el requisito de cabeza de familia para ser nombrado jurado, y, un año después, el Código civil en su artículo rgro hacía responsable al cabeza de familia de las cosas que se cayeren o arrojaren desde la casa en que habita la propia familia (26). Poco tiempo después, la Ley de 29 de junio de I9I I hacía también responsable al cabeza de familia, en determinados casos, del pago del impuesto de inquilinato.

Vemos, pues, por cuantas y distintas necesidades va surgiendo la categoría del cabeza de familia, aunque, si se analizan bien, todas vienen a referirse a la vida local.

En el proyecto Maura, en éste como en otros muchos aspectos, reside el precedente inmediato del Estatuto Municipal de 1924, que fué el primer texto legal que creó el concepto de cabeza de familia en la clasificación de la población municipal. De aquí pasó a la Ley municipal de 1935, al proyecto Iturmendi de 1941 y a la Ley de Bases de I945.

El Estatuto municipal formuló la siguiente definición de cabeza de familia: "Son cabezas de familia los jefes de casa mayores. de edad o emancipados, bajo cuya dependencia en algún modo vivan los individuos de la casa, si los hubiere. Pueden ser o no veci-

(25) Diario de Sesiones de 7 de febrero de 1908, núm. 143, pág. 4.489.

(26) Según Manresa, este precepto tenía ya precedentes en el Derecho Romano (párrafo 1.o, tít. V, del lib. IV de la Instituta) y en las Partidas (Leyes XXV y XXVI del tít. XV de la Part. VII). El proyecto de 1851 se apartó de estos precedentes, que imponian la responsabilidad a todos los que morasen en la casa, para hacer único iesponsable al jefe de la misma o cabeza de familia. Del proyecto de 1851 pasó sin duda al Código civil. 
nos, españoles o extranjeros, varones o hembras" (27). Para el Estatuto, además, "el cabeza de familia es el representante legal de la casa. Como tal, posee los derechos que le reconoce la Ley y podrá ser compelido por la autoridad local a que, bajo su personal responsabilidad, cumplimente los servicios que estime necesarios y legítimamente sean debidos" (28).

Estos derechos son, entre otros, el de sufragio que reconoce el mismo texto legal, en su art.ículo $5^{\mathrm{I}}$, a las mujeres cabezas de familia, precedente indudable de la Ley de Bases de Régimen Local. En cuanto a los servicios que deberá cumplimentar, se expresan en el artículo 30 del Reglamento de Población y términos municipales, todavía vigente.

Aun reconociendo el mérito de la innovación del Estatuto municipal, estimamos, sin embargo, defectuosa la definición que da del cabeza de familia, por la imprecisión de la frase "bajo cuya dependencia convivan los individuos de la casa", pues dentro de este concepto caben los hijos casados y los criados casados y viudos con hijos, que indudablemente constituyen una familia independiente.

Con esto terminamos la primera parte de este trabajo, dedicada al estudio del origen y evolución del concepto de cabeza de familia en España, y pasamos a la segunda, que comprenderá, a su vez, dos puntos: uno, dedicado al análisis y crítica del concento de cabeza de familia en la legislación española vigente, y otro, a senalar criterios prácticos para su determinación.

\section{I I}

El Concepto de cabeza de familia en la legislación española VIGENTE

1) Análisis y crítica del concepto.

La legislación española vigente, en lo que al cabeza de familia se refiere, aparte de lo dispuesto en leyes especiales, la constituyen por orden cronológico:

(27) Artículo 26, que coincide con el 10 del Proyecto Maura de 1907.

(28) Articulo 27, que coincide con el 12 del Proyecto Maura de 1907. 
I. La Ley municipal de 1935 y el Reglamento de Población y Términos Municipales de 1924, en cuanto al concepto y obligaciones del cabeza de familia en los Ayuntamientos.

2. La Instrucción para realizar el Censo de población de 1940, ya que con arreglo a sus normas se realizó la inscripción censal de aquella fecha, hoy vigente, y de la que es copia el Padrón de 1940, base, con sus rectificaciones, del Censo de Cabezas de Familia.

$3 .^{\circ}$ La Ley de Bases de 1945, como inspiradora del Decreto de 29 de septiembre de 1945 , y este Decreto, en todo lo que se réfiere a la Formación del Censo de Cabezas de Familia.

La Ley municipal de 1935 copió literalmente el concepto de cabeza de familia dado en el Estatuto Municipal, si bien corrigiendo la frase que criticamos y sustituyéndola por la de "bajo cuya dependencia vivan en algún modo los individuos de la familia", con lo cual se excluían los criados y demás servidumbre, pero no los familiares, que siendo miembros, como cabezas o no, de otra familia convivan en la casa. Adolece, pues, también de imperfección este concepto.

La Instrucción de 1940 para realizar el Censo de Población contiene una definición, a nuestro parecer muy técnica y perfecta, de lo que debe entenderse por familia. La define como la "reunión de convivientes, presentes o no en el término la noche censal, sometidos cuando menos a la autoridad domiciliaria del cabeza. La forman la cónyuge, los hijos no emancipados y los parientes, criados y allegados que no pertenezcan como cabezas o como sometidos a otra familia en el mismo Municipio" (art. 26). En cambio, formarán familias distintas dentro del mismo domicilio los emancipados que han creado una nueva familia, con propios sometidos, y que son así nuevos cabezas en la comunidad domiciliaria.

Los cabezas de familia, que pueden ser transeúntes y extranjeros, serán los que ejerzan la autoridad familiar; el padre, o en su defecto la madre o persona que asumiera la representación fạmiliar en la vida común (art. 25).

El criterio que inspira este concepto es el de la familia en sentido jurídico estricto. Es decir, que la familia, como afirma CASTÁn y con él otros muchos tratadistas, cabe conceptuarla de dos mane- 
ras, bien en sentido vulgar, entendiendo por tal la "reunión de personas que viven bajo un mismo techo sometidas a la dirección y recursos del jefe de la casa", o bien en sentido jurídico, que puede ser amplio y estricto; amplio, si entendemos por familia el conjunto de personas unidas por el matrimonio o por los vínculos de parentesco (natural y de adopción); o estricto, si sólo se comprende el grupo de personas formado por los cónyuges o por los padres e hijos, con exclusión de los demás parientes, es decir, integrada solamente por relaciones conyugales y paterno-filiales.

En el concepto de la Instrucción, que venimos analizando, se admiten los criados o allegados dentro de la familia, pero siempre que no pertenezcan como cabezas o sometidos a otra familia dentro del mismo Municipio.

Con esto se amplía el sentido jurídico estricto de la familia; sin embargo, este concepto es el que juega para determinar la existencia o no de varias familias en el mismo domicilio, y por ello se dice que formarán familia los emancipados que hayan creado una nueva, o "el matrimonio con o sin hijos" que compartan el domicilio con otra familia. En una palabra, es el matrimonio, con sus relaciones conyugales y paterno-filiales, el criterio base de la distinción de una o varias familias. Criterio éste que estimamos acertado, pues cuando se trata de distinguir si hay una o varias familias no debe acudirse a otras consideraciones (aparte de las ficciones que establezca la ley) que al medio único de constituirse la familia: el matrimonio. Todas las ampliaciones que en distintas leyes y a distintos fines reciba la familia serán siempre meras ficciones de la ley; creaciones artificiosas que podrán ser útiles y convenientes a determinadas finalidades, pero que nunca podrán considerarse como familias en sentido jurídico estricto, si no tienen por base de su constitución el matrimonio.

Y la consecuencia lógica de cuanto venimos exponiendo es que el cabeza de familia será -como dice también la Instrucción censal- el padre, o en su defecto la madre o la persona que asuma su representación.

Fijado así el concepto de familia, pasemos ahora al examen del concepto de cabeza de familia en la novísima legislación española, 
constituída por la Ley de Bases de Régimen Local y el Decreto de 29 de septiembre de 1945 .

Dice así la primera, en su Base $5 .^{\text {a }}$ : "Es cabeza de familia el mayor de edad o emancipado bajo cuya dependencia convivan otras personas en un mismo domicilio."

Es decir, que lo que caracteriza la existencia de un cabeza de familia es que convivan bajo su dependencia otras personas. Se ha querido, indudablemente, ampliar aquí el concepto estricto de familia a que antes hacíamos referencia, pero se suscitan algunos reparos. Puede admitirse que para fines administrativos, fiscales, estadísticos y electorales el concepto de cabeza de familia se extienda, por similitud o ficción legal, a otras personas que no son "strictu sensu" cabeza, como los sacerdotes bajo cuya dependencia convivan otras personas, los hermanos mayores representantes de los menores huérfanos, o simplemente la persona más caracterizada en cualquier otro tipo de convivencia cuasi-familiar. Puede admitirse, decimos, esta ampliación, pero siempre teniendo en cuenta que ninguna de esas personas que conviven con el cabeza ficticio hayan constituído una nueva familia, sean casados o viudos con hijos.

Los criterios de convivencia y dependencia económica y domiciliaria en que se apoya el concepto de la Ley. de Bases no son suficientes.

El Decreto de 29 de septienibre, dado para cumplir lo ordenado por la Ley de Bases, forzosamente había de inspirarse en ella, y, como consecuencia, dice en su artículo $5 .^{\circ}$ que "se considerará con derecho a figurar en el Censo electoral, por su condición de cabeza de familia, a los españoles, vecinos y mayores de 2 I años o emancipados mayores de 18 , varones o mujeres, bajo cuya dependencia convivan otras personas en su mismo domicilio y que figuren inscritos con tal carácter en el Padrón municipal de 1940 o en sus apéndices rectificatorios anuales hasta el 3 I de diciembre de I944, o que posteriormente, y hasta el 30 de septiembre de 1945, sean incluídos con las formalidades legales".

Pero obsérvese que sólo los españoles pueden ser vecinos y todos los emancipados lo son según el artículo $3 \mathrm{I}$ de la Ley municipal vigente y la Base $5 .^{2}$ de la nueva Ley de Régimen local. 
REVL-1946, núm. 26. BULLON RAMIREZ, ANTONIO. EL CONCEPTO ESPAÑOL DE CABEZA DE FAMILIA.

No obstante, creemos que puede completarse la Ley de Bases y el Decreto de 29 de septiembre de 1945, por la referencia que el citado artículo $5 .^{\circ}$ hace al Padrón de 1940, hecho con arreglo a las normas dictadas por la Instrucción censal de aquel año. La Instrucción puede ser aquí la legislación supletoria que ayude en cada caso a determinar prácticamente quiénes tienen o no derecho a figurar en el Censo electoral de Cabezas de Familia.

2) Criterios prácticos para determinar, con arreglo a la legislación vigente, quiénes deben tener la consideración de electores por ser cabezas de familia.

Si se armoniza toda esta legislación que hemos ido analizando y se utiliza con buen criterio, creemos que con referencia a ella puede determinarse en cada caso concreto y con arreglo a la misma el otorgamiento o no del derecho de sufragio. Como reglas prácticas para determinarlo podemos señalar las siguientes:

I. $^{2}$ Hay que tener presente siempre si el concepto de cabeza de familia va a ser fijado para fines electorales o para fines estadísticos o administrativos. En el primer caso, hay que excluir a todos los extranjeros y transeúntes, que no pueden ser electores y pueden ser cabezas de familia a otros fines y figurar con tal carácter en el Padrón.

2. ${ }^{\mathrm{a}} \quad$ No basta la simple convivencia unida a la dependencia económica para señalar uno o varios cabezas en el mismo domicilio. Hay que acudir al estado civil de los sometidos, pues si fueren casados o viudos con hijos deberán ser electores si, además, figuran como cabezas de familia en el Padrón.

$3 .^{a}$ En el caso de convivencia cuasi-familiar de varias personas, hermanos, amigos, etc., que no sean a su vez cabezas, con arreglo a la regla anterior, puede considerarse como cabeza y elector al más caracterizado de ellos, guiándose, para determinarlo, por sig. nos externos, como ser el dueño de la vivienda, tener a su nombre el contrato de inquilinato, etc.

$4 .^{2}$ Si habitase una persona completamente sola en una vivienda, sin servidumbre ni allegados de ninguna clase, no debe conside- 
rarse ni cabeza de familia ni elector, ya que no convive nadie bajo su dependencia, y

5. ${ }^{\text {a }}$ Si hubiera menores no emancipados viviendo separadamente del tutor y bajo cuya dependencia convivan otras personas, como hermanos menores, servidumbre, etc., tampoco podrán ser considerados como cabezas ni como electores por no ser vecinos.

No obstante, para fines puramente estadísticos cabría considerarlos como cabezas, pues nada se opone, por ejemplo, a que un menor no emancipado llene su cédula en la inscripción censal.

Antonio Bullón Ramf́rez 